\title{
Circadian rhythm in the assessment of postconcussion insomnia: a cross-sectional observational study
}

\author{
Dora M. Zalai MD PhD, Todd A. Girard PhD, Michael D. Cusimano MD PhD, \\ Colin M. Shapiro MBBCh PhD
}

Abstract

Background: Insomnia is a major predictor of adverse outcomes in mild traumatic brain injury (mTBI), including concussion; although insomnia symptoms may be due to various sleep disorders, those related to circadian rhythm sleep-wake disorders (CRSWDs) require specific assessment and treatment. The objective of the current study was to determine the prevalence of CRSWD in a sample of treatment-seeking people with chronic insomnia symptoms after an mTBI.

Methods: Participants aged 17-65 years who had experienced an mTBI and reported chronic insomnia were recruited from diverse community clinics in Ontario 3-24 months after their injury to participate in this cross-sectional observational study. Potential participants were screened by both telephone and intake interview. Exclusion criteria were alcohol or substance use disorders, preexisting brain disorder or previous neurosurgery, recent travel across more than 2 time zones or shift work. Assessments included a clinical interview, questionnaires, 2 weeks of actigraphy and a sleep diary, and a dim-light melatonin onset test. The main outcome measure was the proportion of patients with CRSWDs.

Results: Of the 50 participants (32 [64\%] female; median age $39.5 \mathrm{yr}), 13$ (26\% [standard deviation 12\%]) had an CRSWD. The most common circadian diagnosis was delayed sleep-wake phase disorder (10 participants [20\%]).

Interpretation: The prevalence of CRSWDs may be exceptionally high among people with chronic insomnia symptoms following mTBI. Proper detection and treatment of CRSWDs in this population is essential to facilitate recovery. The findings emphasize the relevance of a diagnostic circadian assessment in patients with mTBI presenting with chronic insomnia symptoms.

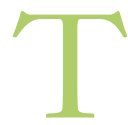
raumatic brain injury (TBI) is the leading cause of disability among young adults, and the incidence is increasing in Canada. ${ }^{1,2}$ Mild TBI (mTBI), including concussion, accounts for the majority $(70 \%-90 \%)$ of treated TBI cases and has the largest contribution to injuryrelated disability. ${ }^{3}$ Early detection and effective management of medical conditions that hinder recovery could prevent disability and substantially reduce societal costs.

In this regard, sleep disruptions are key modifiable targets that magnify the sequelae of mTBI. ${ }^{4}$ Insomnia - difficulties falling or staying asleep, or early-morning final awakening — is the most common persistent sleep symptom after $\mathrm{mTBI}^{5,6}$ and is particularly prevalent following mTBI compared to more severe forms. ${ }^{4}$ Moreover, insomnia after concussion worsens fatigue, pain, cognition and mood, and predicts poor overall prognosis for recovery, greater disability and mental disorders. ${ }^{7-11}$

Given that disorders of sleep and wakefulness can be treated effectively, treatment holds the promise of improving the management of persistent postconcussive symptoms and hastening recovery from mTBI. Recent reports ${ }^{4,12,13}$ emphasize the need for multimodal sleep and circadian assessments; however, these are not common practice, and research remains limited.

Standard assessment of insomnia relies on patients' subjective report, and the recommended first-line treatment is cog- nitive behavioural therapy. ${ }^{14,15}$ Importantly, however, circadian rhythm sleep-wake disorders (CRSWDs) that may underlie symptoms of insomnia require fundamentally different assessment and treatment (Appendix 1, available at www. cmajopen.ca/content/8/1/E142/suppl/DC1). These disorders arise if there is a disruption of the endogenous circadian system or a misalignment between the internal circadian rhythm and the external environment. The best biologic marker of CRSWDs is abnormal dim-light onset of melatonin production, and the behavioural diagnostic feature is that the sleep phase is markedly delayed, advanced or irregular relative to environmental time and social norms ${ }^{16}$ (Appendix 1).

Rates and mechanisms of CRSWDs in mTBI are not well characterized, but evidence indicates that brain trauma can disrupt systems that regulate circadian rhythms, such as suprachiasmatic nucleus function, gene expression, neural plasticity,

Competing interests: None declared.

This article has been peer reviewed.

Correspondence to: Dora Zalai, drzalai@sleep-psychology.ca

CMAJ Open 2020. DOI:10.9778/cmajo.20190137 
and melatonin production and transmission..$^{4,17,18}$ For instance, mTBI may cause pineal gland injury and disrupt circadian regulation via its effect on melatonin secretion. ${ }^{19}$

To our knowledge, only 1 clinical study has examined diagnostic circadian assessment in patients with mTBI. ${ }^{20} \mathrm{~A}$ high prevalence of CRSWDs was found among participants.

Given that CRSWDs require specific circadian assessment and treatment, it is pertinent to establish whether patients with $\mathrm{mTBI}$ and insomnia symptoms constitute one of the few clinical groups in which CRSWDs are prevalent. If so, circadian assessment and treatment becomes an important part of chronic insomnia investigation and management following mTBI.

The objective of the current study was to determine the prevalence of CRSWDs (according to standard diagnostic criteria using evidence-based comprehensive assessment including actigraphy and dim-light melatonin onset) in a sample of treatment-seeking people with chronic insomnia following mTBI. Our hypothesis was that CRSWDs are more common in this clinical group than among people who seek treatment for chronic insomnia in sleep clinics. ${ }^{16}$

\section{Methods}

This was a cross-sectional observational study. Data were collected at a sleep (Sleep and Alertness Clinic) and a melatonin testing laboratory in Toronto in 2016 and 2017, and were analyzed in 2018.

\section{Participants}

Information about the study was distributed widely to hospitals and community clinics in southern Ontario (primarily the Greater Toronto Area) and lower regions of northern Ontario, posted on brain injury and sleep clinic websites, and disseminated by word of mouth. Referral sources included a range of clinics and specialists.

Participants were included if they had had an mTBI (Glasgow Coma Scale score $\geq 13$ at the time of injury) 3-24 months before the screening assessment, had chronic insomnia symptoms that started or amplified markedly after the injury, and were aged 17-65 years. If a participant had multiple concussions, we calculated time elapsed since the injury that preceded the onset of the sleep problem (3-24 mo). Participants were excluded if they had alcohol or other substance use problems within 3 months before enrolment based on the criteria of the fifth edition of the Diagnostic and Statistical Manual of Mental Disorders (DSM-5), needed immediate psychiatric help based on in-person assessment, had preexisting brain disorders or had had neurosurgery, had travelled across more than 2 time zones less than 3 weeks before the assessment or had a shift work schedule.

\section{Procedures}

Prospective participants were screened initially with a telephone interview (5-10 min). The telephone screening assessment included questions about the presence of insomnia symptoms, history of brain injury, preexisting brain disorder, age, substance use, travel across time zones and shift work history. Those who passed the telephone screen participated in an in-person assessment that included a standard interview and completion of questionnaires (90-120 min). When family members accompanied participants, they were present during the interview and provided collateral information with participants' consent.

In the 12-14 days after their enrolment, participants kept a sleep diary and wore a Philips Respironics Actiwatch on their nondominant wrist. They participated in a dim-light melatonin onset test 12-14 days after their initial interview.

\section{Assessment tools and methods}

\section{Insomnia assessment}

The Duke Structured Interview for Sleep Disorders assesses sleep disorders according to clinical and research diagnostic criteria. ${ }^{21}$ We used an updated version of this tool in which the insomnia section has been modified to match the new DSM-5 criteria to select participants with chronic insomnia.

The Insomnia Severity Index measures the subjective severity of insomnia symptoms..$^{22,23}$ The degree of insomnia severity is determined by the summary scores. A summary score of 11 has been recommended as a cut-off for screening for clinical insomnia research ${ }^{24}$ and was used as cut-off for inclusion in the current study.

\section{Assessment of sleep pattern}

Wrist actigraphy is a recommended objective diagnostic assessment tool for CRSWDs. ${ }^{16}$ Participants wore a Philips Respironics Actiwatch 2 on their nondominant wrist. The device detects movement by means of a solid piezoelectric accelerometer with $0.35-$ to $7.5-\mathrm{Hz}$ bandwidth and $0.5-2 \mathrm{G}$ peak value. The device was set to record 30 -second epochs at medium sensitivity to detect wake threshold. The actigraphy recording showed the regularity and timing of sleep periods, and the Actiware 6.0.7 software calculated all the sleep parameters, including sleep onset latency, wake after sleep onset, total sleep time and sleep efficiency.

The Consensus Sleep Diary was developed to provide a standard sleep log based on patients' input and expert consensus. ${ }^{25}$ The sleep indices in the diary differentiate between people with insomnia and good sleepers. ${ }^{26} \mathrm{We}$ used the diary for longitudinal subjective assessment of sleep pattern.

\section{Biologic assessment of circadian phase}

The dim-light melatonin onset test (Saliva Melatonin ELISA [enzyme-linked immunosorbent assay] kit [EK-DSM], Bühlmann Laboratories) comprises 8 saliva samples collected according to standard procedures. Three baseline samples were taken every 30 minutes, and the remaining samples were collected hourly. Data collection started 6 hours before habitual bedtime, which was determined based on participants' self-report and was verified from their sleep diary data. Medications that influence melatonin levels were prohibited or discontinued before the test.

Saliva samples were frozen and analyzed by means of ELISA as per the manufacturer's instructions. The intra- and interassay coefficients of variation were 7.62 and 8.88 , respectively. 
As per consensus guidelines, ${ }^{27}$ the time of dim-light melatonin onset was determined as the time at which the melatonin concentration reached and remained above the threshold (2 standard deviations above the baseline or, in cases in which there were fewer than 3 baseline values, an absolute value of $3 \mathrm{pg} / \mathrm{mL})$.

\section{Diagnosis of circadian rhythm sleep-wake disorder}

We diagnosed CRSWDs according to the third edition of the International Classification of Sleep Disorders ${ }^{16}$ based on agreement between a physician and a clinical psychologist (C.M.S. and D.M.Z.), both with specialization in sleep medicine. All 3 assessors were blind to the study hypothesis with the exception of the first author (D.M.Z.), who was involved in the administration of aspects of the assessment.

\section{Statistical analysis}

Based on the rate of CRSWDs in mTBI of $36 \%$ observed by Ayalon and colleagues, ${ }^{20}$ an upper rate of CRSWDs generally associated with insomnia of $10 \%,{ }^{16} 2$-tailed $\alpha=0.05$ and $95 \%$ power, we set 34 participants as a lower limit for recruitment to address our hypothesis. ${ }^{28}$

We compared the observed proportion of CRSWDs with the population estimate of $10 \%^{16}$ using the $z$ test for 1 proportion, and $95 \%$ confidence intervals (CIs) for the prevalence rates were based on the corresponding precision afforded by our final sample size $(n=50) .^{29}$

\section{Ethics approval}

This study was approved by the Ryerson University Research Ethics Board.

\section{Results}

Participant selection is summarized in Figure 1. The final study group comprised 50 patients with mTBI and chronic insomnia symptoms. Participant characteristics and referral sources are summarized in Table 1.

Two participants had missing actigraphy data, and therefore we used their sleep diary data to determine whether they had a normal sleep phase. Three participants did not complete the dim-light melatonin onset test, and results could not be determined precisely from 5 samples (the onset was outside the measurement period in 3, and there were technical problems with 2 samples; these 2 were not included in the analysis).

Most participants (46 [92\%]) had moderate or severe insomnia symptoms (Insomnia Severity Index score $\geq 15$ ). As reported during the interviews and verified by family when present, 37 participants (74\%) did not have subjective sleep problems or sleep disorders before their injury.

Thirteen participants (26\% [standard deviation 12\%]) received a diagnosis of CRSWD, which supports our hypothesis of a greater proportion than the expected base rate of $10 \%$ for more general insomnia samples $(z=3.77, p<0.001)$. The most common diagnosis was delayed sleep-wake phase disorder (10 patients [20\%]). The mean time at dim-light melatonin onset for these patients was 2253 (95\% CI 2218-

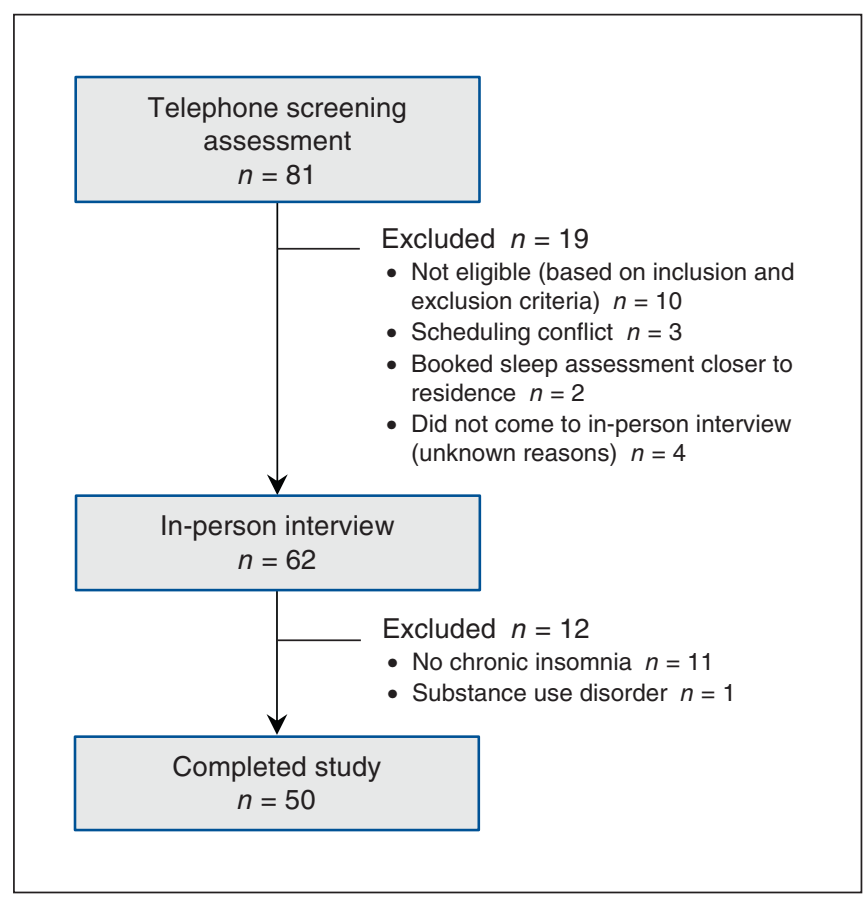

Figure 1: Flow diagram showing participant selection.

2318), which represented a significant delay compared to those without a CRSWD (mean 2024, 95\% CI 2006-2040, $t=4.22$, 32 degrees of freedom, $r=0.73$ ).

The median age of those with delayed sleep-wake phase disorder was 26 years (interquartile range [IQR] 20-30 yr); only 1 of these participants was older than 32 years. There were 2 participants with advanced sleep-wake phase disorder and 1 participant with irregular sleep-wake rhythm disorder; all 3 were more than 40 years. The median age of the 37 participants without CRSWDs was 40 years (IQR 31-54 yr).

There was no meaningful difference in the average Insomnia Severity Index score between participants with normally entrained circadian rhythm (19.8), those who had delayed sleep-wake phase disorder (20.7) and those who had advanced sleep-wake phase disorder (20.5). The Insomnia Severity Index score of the single participant with an irregular sleepwake rhythm disorder was 24 .

\section{Interpretation}

We carried out a rigorous multimethod diagnostic circadian assessment based on standard criteria in a representative sample of patients with mTBI and chronic insomnia. The results support our hypothesis: $26 \%$ of the sample received a diagnosis of CRSWD. Previous circadian studies have included patients with moderate and severe TBI, assessed melatonin secretion without determining dim-light melatonin onset, or determined average melatonin onset in a group of patients without providing diagnostic assessment and interpretation, in samples that were substantially smaller than the current sam-

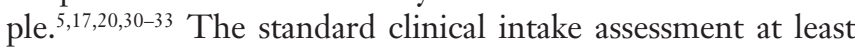
3 months after the TBI ensured that a sample of people with chronic insomnia symptoms was included. 


\begin{tabular}{|c|c|}
\hline Characteristic & $\begin{array}{c}\text { No. }(\%) \text { of participants }{ }^{*} \\
n=50\end{array}$ \\
\hline \multicolumn{2}{|l|}{ Age, yr } \\
\hline Median (IQR) & $39.5(23.8)$ \\
\hline Range & $17-62$ \\
\hline \multicolumn{2}{|l|}{ Gender } \\
\hline Male & $18(36)$ \\
\hline Female & $32(64)$ \\
\hline \multicolumn{2}{|l|}{ Education } \\
\hline High school & $13(26)$ \\
\hline College & $12(24)$ \\
\hline University & $25(50)$ \\
\hline \multicolumn{2}{|l|}{ Employment status } \\
\hline Full-time & $21(42)$ \\
\hline Part-time & $5(10)$ \\
\hline Student & $4(8)$ \\
\hline Unemployed & $8(16)$ \\
\hline Sick leave/disability & $11(22)$ \\
\hline Retired & $1(2)$ \\
\hline \multicolumn{2}{|l|}{ No. of prior concussions } \\
\hline 0 & $32(64)$ \\
\hline$\geq 1$ & $18(36)$ \\
\hline \multicolumn{2}{|l|}{ Time since injury, mo† } \\
\hline Median (IQR) & $11.5(12)$ \\
\hline Range & $3-24$ \\
\hline \multicolumn{2}{|l|}{ Cause of injury } \\
\hline Car accident & $18(36)$ \\
\hline Sports injury & $18(36)$ \\
\hline Fall & $9(18)$ \\
\hline Hit on head by object & $2(4)$ \\
\hline Physical assault & $2(4)$ \\
\hline Workplace injury & $1(2)$ \\
\hline Comorbidities $\ddagger$ & $22(44)$ \\
\hline Chronic pain & $13(26)$ \\
\hline Hypertension & $5(10)$ \\
\hline $\begin{array}{l}\text { Gastrointestinal disease } \\
\text { (e.g., gastrointestinal reflux) }\end{array}$ & $5(10)$ \\
\hline Hyperlipidemia & $3(6)$ \\
\hline Asthma & $3(6)$ \\
\hline Autoimmune disease & $2(4)$ \\
\hline Hypothyroidism§ & $2(4)$ \\
\hline Osteoporosis & $1(2)$ \\
\hline Heart disease & $1(2)$ \\
\hline Past psychiatric condition & $23(46)$ \\
\hline
\end{tabular}

\begin{tabular}{|c|c|}
\hline Characteristic & $\begin{array}{c}\text { No. }(\%) \text { of participants } \\
n=50\end{array}$ \\
\hline Current psychiatric condition & $13(26)$ \\
\hline Major depressive disorder & $7(14)$ \\
\hline Social anxiety disorder & $4(8)$ \\
\hline Panic disorder & $2(4)$ \\
\hline Obsessive-compulsive disorder & $1(2)$ \\
\hline Specific phobia & $1(2)$ \\
\hline \multicolumn{2}{|l|}{ Referral source } \\
\hline Head injury clinic & $18(36)$ \\
\hline Family medicine clinic & $16(32)$ \\
\hline Concussion/sports clinic & $6(12)$ \\
\hline Sleep clinic & $4(8)$ \\
\hline $\begin{array}{l}\text { Speech-language pathologist, } \\
\text { neurologist, physiotherapist }\end{array}$ & $4(8)$ \\
\hline Emergency specialist & $2(4)$ \\
\hline \multicolumn{2}{|c|}{$\begin{array}{l}\text { Note: IQR = interquartile range. } \\
\text { *Except where noted otherwise. } \\
\text { tIn participants with multiple concussions, time elapsed since the injury that } \\
\text { preceded the onset of the sleep problem. } \\
\text { fSome participants had multiple comorbidities. } \\
\text { §Participants who reported a history of hypothyroidism were using thyroid } \\
\text { supplements. }\end{array}$} \\
\hline
\end{tabular}

Ayalon and colleagues ${ }^{20}$ selected 15 participants from among 42 sleep clinic patients with mTBI and insomnia. A CRSWD was diagnosed in all 15 participants, which suggests that the prevalence of CRSWDs may be higher among patients with mTBI who have with chronic insomnia symptoms than in relevant comparison groups (i.e., sleep clinic patients with insomnia). However, selection criteria, dim-light melatonin onset and time since injury were not specified, and the age range was restricted.

Most (77\%) of the observed CRSWDs in our study reflected delayed sleep-wake phase disorder. The observed proportion of participants with delayed sleep-wake phase disorder, $20 \%$, is $1-2$ orders of magnitude higher than in the general population $(0.17 \%-1.53 \%)$ and over 3 times that among patients with chronic insomnia treated at sleep clinics $(6.7 \%) .{ }^{34-36}$ Estimates for adolescents are $3 \%-7 \%$ in the general population and $7 \%-16 \%$ in clinical samples of people with psychiatric conditions. ${ }^{16,37-39}$ The particularly high prevalence in the younger mTBI participants in the current study underscores the need for more studies to determine the rates of CRSWDs across demographic and clinical samples.

This study did not explore the causes of delayed sleepwake phase disorder, but several etiologic factors merit consideration. The authors of a magnetic resonance imaging study noted that patients who had sleep problems after an mTBI had a longer cerebellar tentorium and a flatter tentorial angle than patients who did not have sleep problems. ${ }^{19}$ They speculated that the pineal gland (the site of melatonin 
secretion) would be affected by injuries caused by an anteroposterior force in people with this anatomic predisposition.

Another biologic predisposing factor could be a susceptibility of the sleep and circadian system for phase delay owing to a long circadian period, heightened responsiveness to the melatonin-suppressing effect of light in the evening, or slow accumulation and dissipation of homeostatic sleep drive. ${ }^{40}$ If the social demand to wake up early in the morning lessens during the postinjury recovery period, the sleep phase follows the circadian signals and shifts to later hours in people with a predisposition for phase delay. This could lead to a vicious cycle in which longer light exposure in the evening and lack of light exposure in the morning further delay the circadian cycle. ${ }^{41}$

It is possible that people with delayed dim-light melatonin onset or evening chronotype before their injury are at increased risk for a TBI because they are sleepy in the morning when they attend sport training, school or work. Athletic performance fluctuates across the circadian cycle, and excessive sleepiness affects cognitive performance and increases the risk for accidents and injuries. ${ }^{42-48}$ Thus, such susceptibility factors may partly contribute to a high prevalence of delayed sleep-wake phase disorder in mTBI.

Two participants in our study received a diagnosis of advanced sleep-wake phase disorder. Reports of this disorder are rare, including only individual cases and family cohorts. ${ }^{16,35}$ In the current study, neither of the participants with the disorder had a family history of this condition.

One participant was diagnosed with irregular sleep-wake rhythm disorder. This CRSWD has previously been described among people with brain disorders. ${ }^{16}$ The affected person in the present study had an alcohol use disorder until 6 months before the injury. There is an interaction between the circadian system and alcohol use, and it is possible that preinjury alcohol use disorder increases the risk of an irregular sleep-wake rhythm disorder following a brain injury. ${ }^{49}$

Awareness of CRSWDs is generally low among clinicians, but distinguishing them from insomnia disorder is crucial since the treatment of insomnia disorder (cognitive behavioural therapy or selected hypnotic medications) and the management of CRSWDs (melatonin and bright light therapy) are fundamentally different. ${ }^{14,50}$

Our results indicate that clinicians should be attentive to symptoms of CRSWDs when they see patients with persistent insomnia symptoms following an mTBI, including concussion. Referral for a circadian assessment is warranted if there is a marked shift of the sleep period or if the patient sleeps only for short (maximum 4-hr) periods around the clock. ${ }^{18,50}$ We suggest that the threshold for referring to a circadian assessment should be especially low if a teenager or young adult reports chronic sleep-onset insomnia or a delay of sleep phase following an mTBI.

The results of the current study highlight the need to assess CRSWDs in patients with chronic insomnia following an mTBI. Circadian assessment is currently not part of a standard investigation of postconcussion insomnia and is not a routine component of sleep clinic assessments. Consequently, based on our results, about $25 \%$ of patients with symptoms of postconcussion chronic insomnia would not receive an appropriate diag- nosis and thereby tailored treatment for their sleep difficulty, which, in turn, would lead to lower likelihood of recovery. ${ }^{6-8}$ Future longitudinal studies should identify factors that predispose people to a CRSWD after a concussion, and test the nature and direction of pathways between the circadian timing of sleep and wakefulness and factors that may influence this relation in mTBI in various age groups. Given the targeted selection process for this study, future research should aim to ascertain rates of CRSWDs through broader recruitment of mTBI populations. In addition, future studies should assess CRSWDs in larger samples of people with mTBI who have insomnia.

Replication of this study will benefit from inclusion of an age- and sex-matched comparison group of patients with chronic insomnia who did not have a history of TBI to allow for more direct comparison of rates of CRSWDs and sleepwake measures. Given that research on CRSWDs among patients with insomnia alone is limited, future studies that include comparison groups will add valuable information not only for those with TBI but also to the general insomnia and CRSWD literature.

Of potential interest for future study, the median age of those with delayed sleep-wake phase disorder in the current study was 26 years (IQR 20-30 yr); only 1 of these people was older than 32 years.

\section{Limitations}

Some limitations of our study should be considered. First, the sample may overrepresent patients who are distressed by their insomnia symptoms. At the same time, these are the patients who (ideally) would receive some form of postconcussion sleep assessment in the community. Second, we did not recruit people with substance use disorders or shift workers. It is likely that the frequency of CRSWDs is higher in these groups than in our sample. Also, participants may not have reported insomnia symptoms that predated their injury. This does not bias the diagnoses but precludes inferences about the possible role of the injury. The results pertain to treatmentseeking people with chronic insomnia symptoms who had an mTBI 3-24 months before the sleep assessment and may not be generalizable beyond this group. Although sufficient for our current aims, the sample size limited precision in the estimated prevalence of CRSWDs. In addition, we did not include an age- and sex-matched comparison group of patients with chronic insomnia who did not have a history of TBI. The current study did not aim to establish a cause-andeffect relation between the clinical diagnoses and the injury, or control for variables that may have influenced this relation.

\section{Conclusion}

A CRSWD was diagnosed in about one-quarter of treatmentseeking people with symptoms of postconcussion chronic insomnia. This suggests that clinicians may include CRSWDs in their diagnostic algorithm and consider the potential benefits of a circadian assessment when patients report insomnia symptoms and a marked change in their sleep phase following an mTBI. The majority of participants in whom a CRSWD was diagnosed were adolescents and young adults with a 
delayed phase sleep-wake disorder; this finding warrants larger-scale replication. Given that insomnia symptoms are major predictors of poor postconcussion recovery, detection and effective management of CRSWDs may improve recovery in those who experience insomnia following an mTBI.

\section{References}

1. Rao DP, McFaull S, Thompson W, et al. Trends in self-reported traumatic brain injury among Canadians, 2005-2014: a repeated cross-sectional analysis. CMA7 Open 2017;5:301-7.

2. Neurological disorders: public health challenges. Geneva: World Health Organization; 2006

3. Cassidy JD, Carroll LJ, Peloso PM, et al. Incidence, risk factors and prevention of mild traumatic brain injury: results of the WHO Collaborating Centre Task Force on Mild Traumatic Brain Injury. 7 Rehabil Med 2004;(Suppl 43):28-60.

4. Wickwire EM, Schnyer DM, Germain A, et al. Sleep, sleep disorders, and circadian health following mild traumatic brain injury in adults: review and research agenda. 7 Neurotrauma 2018;35:2615-31.

5. Grima N, Ponsford J, Rajaratnam SM, et al. Sleep disturbances in traumatic brain injury: a meta-analysis. 7 Clin Sleep Med 2016;12:419-28.

6. Mathias JL, Alvaro PK. Prevalence of sleep disturbances, disorders, and problems following traumatic brain injury: a meta-analysis. Sleep Med 2012;13:898-905.

7. Mollayeva T, Shapiro CM, Mollayeva S, et al. Modeling community integration in workers with delayed recovery from mild traumatic brain injury. $B M C$ Neurol 2015;15:194.

8. Theadom A, Cropley M, Parmar P, et al. Sleep difficulties one year following mild traumatic brain injury in a population-based study. Sleep Med 2015;16:926-32.

9. Theadom A, Starkey N, Jones K, et al. Sleep difficulties and their impact on recovery following mild traumatic brain injury in children. Brain Inj 2016;30:1243-8.

10. Mollayeva T, Pratt B, Mollayeva S, et al. The relationship between insomnia and disability in workers with mild traumatic brain injury/concussion: insomnia and disability in chronic mild traumatic brain injury. Sleep Med 2016;20:157-66.

11. Mollayeva T, Mollayeva S, Shapiro CM, et al. Insomnia in workers with delayed recovery from mild traumatic brain injury. Sleep Med 2016;19:153-61.

12. Mollayeva T, Colantonio A, Mollayeva S, et al. Screening for sleep dysfunction after traumatic brain injury. Sleep Med 2013;14:1235-46.

13. Wickwire EM, Williams SG, Roth T, et al. Sleep, sleep disorders, and mild traumatic brain injury. What we know and what we need to know: findings from a national working group. Neurotherapeutics 2016;13:403-17.

14. Qaseem A, Kansagara D, Forciea MA, et al. Management of chronic insomnia sisorder in adults: a clinical practice guideline from the American College of Physicians. Ann Intern Med 2109;165:125-33

15. Wilson SJ, Nutt DJ, Alford C, et al. British Association for Psychopharmacology consensus statement on evidence-based treatment of insomnia, parasomnias and circadian rhythm disorders. 7 Psychopharmacol 2010;24:1577-601.

16. International classification of sleep disorders: diagnostic and coding manual. 3rd ed. Westchester (IL): American Academy of Sleep Medicine; 2014.

17. Shekleton JA, Parcell DL, Redman JR, et al. Sleep disturbance and melatonin levels following traumatic brain injury. Neurology 2010;74:1732-8.

18. Sabir M, Gaudreault PO, Freyburger M, et al. Impact of traumatic brain injury on sleep structure, electrocorticographic activity and transcriptome in mice. Brain Behav Immun 2015;47:118-30.

19. Yaeger K, Alhilali L, Fakhran S. Evaluation of tentorial length and angle in sleep-wake disturbances after mild traumatic brain injury. A7R Am 7 Roentgenol 2014;202:614-8.

20. Ayalon L, Borodkin K, Dishon L, et al. Circadian rhythm sleep disorders following mild traumatic brain injury. Neurology 2007;68:1136-40.

21. Edinger JD, Kirby A, Lineberger M, et al. The DUKE structured interview for sleep disorders. Durham (NC): Veterans Affairs and Duke University Medical Centers; 2006.

22. Buysse DJ, Ancoli-Israel S, Edinger JD, et al. Recommendations for a standard research assessment of insomnia. Sleep 2006;29:1155-73.

23. Morin CM. Insomnia: psychological assessment and management. New York: Guilford Press; 1993.

24. Morin CM, Belleville G, Belanger L, et al. The Insomnia Severity Index: psychometric indicators to detect insomnia cases and evaluate treatment response. Sleep 2011;34:601-8.

25. Carney CE, Buysse DJ, Ancoli-Israel S, et al. The consensus sleep diary: standardizing prospective sleep self-monitoring. Sleep 2012;35:287-302.

26. Maich KH, Lachowski AM, Carney CE. Psychometric properties of the consensus sleep diary in those with insomnia disorder. Behav Sleep Med 2018;16:117-134.

27. Benloucif S, Burgess HJ, Klerman EB, et al. Measuring melatonin in humans. $\mathcal{f}$ Clin Sleep Med 2008;4:66-9.

28. Faul F, Erdfelder E, Lang AG, et al. G*Power 3: a flexible statistical power analysis program for the social, behavioral, and biomedical sciences. Behav Res Methods 2007;39:175-91.

29. Naign L, Winn T, Rusili BN. Practical issues in calculating the sample size for prevalence studies. Arch Orofacial Sci 2006;1:9-14.
30. Paparrigopoulos T, Melissaki A, Tsekou H, et al. Melatonin secretion after head injury: a pilot study. Brain Inj 2006;20:873-8.

31. Seifman MA, Adamides AA, Nguyen PN, et al. Endogenous melatonin increases in cerebrospinal fluid of patients after severe traumatic brain injury and correlates with oxidative stress and metabolic disarray. 7 Cereb Blood Flow Metab 2008;28:684-96.

32. Guaraldi P, Sancisi E, La Morgia C, et al. Nocturnal melatonin regulation in post-traumatic vegetative state: A possible role for melatonin supplementation? Chronobiol Int 2014;31:741-5.

33. Steele DL, Rajaratnam SM, Redman JR, et al. The effect of traumatic brain injury on the timing of sleep. Chronobiol Int 2005;22:89-105.

34. Weitzman ED, Czeisler CA, Coleman RM, et al. Delayed sleep phase syndrome. A chronobiological disorder with sleep-onset insomnia. Arch Gen Psychiatry 1981;38:737-46.

35. Schrader H, Bovim G, Sand T. The prevalence of delayed and advanced sleep phase syndromes. 7 Sleep Res 1993;2:51-5.

36. Paine SJ, Fink J, Gander PH, et al. Identifying advanced and delayed sleep phase disorders in the general population: a national survey of New Zealand adults. Chronobiol Int 2014;31:627-36.

37. Lovato N, Gradisar M, Short M, et al. Delayed sleep phase disorder in an Australian school-based sample of adolescents. 7 Clin Sleep Med 2013;9:939-44.

38. Saxvig IW, Pallesen S, Wilhelmsen-Langeland A, et al. Prevalence and correlates of delayed sleep phase in high school students. Sleep Med 2012;13:193-9.

39. Sivertsen B, Pallesen S, Stormark KM, et al. Delayed sleep phase syndrome in adolescents: prevalence and correlates in a large population-based study. $B M C$ Public Health 2013;13:1163.

40. Micic G, Lovato N, Gradisar M, et al. The etiology of delayed sleep phase disorder. Sleep Med Rev 2016;27:29-38.

41. Burgess HJ, Eastman CI. A late wake time phase delays the human dim light melatonin rhythm. Neurosci Lett 2006;395:191-5.

42. Atkinson G, Reilly T. Circadian variation in sports performance. Sports Med 1996;21:292-312.

43. Milewski MD, Skaggs DL, Bishop GA, et al. Chronic lack of sleep is associated with increased sports injuries in adolescent athletes. 7 Pediatr Orthop 2014;34:129-33.

44. Dewald JF, Meijer AM, Oort FJ, et al. The influence of sleep quality, sleep duration and sleepiness on school performance in children and adolescents: a meta-analytic review. Sleep Med Rev 2010;14:179-89.

45. Drake C, Roehrs T, Breslau N, et al. The 10-year risk of verified motor vehicle crashes in relation to physiologic sleepiness. Sleep 2010;33:745-52.

46. Melamed S, Oksenberg A. Excessive daytime sleepiness and risk of occupational injuries in non-shift daytime workers. Sleep 2002;25:315-22.

47. Silva EJ, Wang W, Ronda JM, et al. Circadian and wake-dependent influences on subjective sleepiness, cognitive throughput, and reaction time performance in older and young adults. Sleep 2010;33:481-90.

48. Bioulac S, Micoulaud-Franchi JA, Arnaud M, et al. Risk of motor vehicle accidents related to sleepiness at the wheel: a systematic review and meta-analysis [published erratum in Sleep 2018;41(7)]. Sleep 2017;40(10).

49. Rosenwasser AM. Chronobiology of ethanol: animal models. Alcohol 2015;49: 311-9.

50. Auger RR, Burgess HJ, Emens JS, et al. Clinical Practice Guideline for the Treatment of Intrinsic Circadian Rhythm Sleep-Wake Disorders: advanced sleep-wake phase disorder (ASWPD), delayed sleep-wake phase disorder (DSWPD), non24-hour sleep-wake rhythm disorder (N24SWD), and irregular sleep-wake rhythm disorder (ISWRD). An update for 2015: an American Academy of Sleep Medicine Clinical Practice Guideline. 7 Clin Sleep Med 2015;11:1199-236.

Affiliations: Department of Psychology (Zalai, Girard), Ryerson University, Toronto, Ont.; Oakville Centre for Cognitive Therapy (Zalai), Oakville, Ont.; Division of Neurosurgery and Injury Prevention (Cusimano), St. Michael's Hospital, University of Toronto; Department of Psychiatry (Shapiro), University of Toronto; Youthdale Child and Adolescent Sleep Centre (Shapiro), Toronto, Ont.

Contributors: Dora Zalai conceived the study. Dora Zalai and Colin Shapiro acquired the data, and Dora Zalai and Todd Girard analyzed the data. Dora Zalai drafted the manuscript. All of the authors contributed to the study design, data interpretation and revising the manuscript critically for important intellectual content, approved the final version to be published and agreed to be accountable for all aspects of the work.

Funding: Dora Zalai's research was supported by a Canadian Institutes of Health Research (CIHR) Frederick Banting and Charles Best Canada Graduate Scholarship Award and the Youthdale Foundation. Michael Cusimano's work was supported by CIHR Strategic Team Grant in Applied Injury Research TIR-103946 and the Ontario Neurotrauma Foundation.

Supplemental information: For reviewer comments and the original submission of this manuscript, please see www.cmajopen.ca/content/8/1/ E142/suppl/DC1. 